patients in The Netherlands: a nationwide prospective study. J Infect Dis 2000;182:1291-1299.

\section{Rotavirus Outbreak Associated With Toys}

Rogers and colleagues from Memorial Sloan-Kettering Cancer Center in New York City report a rotavirus outbreak in the 42-bed pediatric unit having both bone marrow transplant patients and non-bone marrow transplant oncology patients.

Nosocomially acquired rotavirus was defined as diarrhea, vomiting, or gastrointestinal upset with onset 48 hours or more after hospital admission, accompanied by a positive enzyme immunoassay for rotavirus antigen. Between February 24 and April 4, 1997, eight patients on the pediatric unit had nosocomial rotavirus. Aggressive infection control measures were instituted. Patients with rotavirus were cohorted and placed on Contact Precautions (strict hand washing, gloves, and gown). Investigation by the infection control team revealed that communal toys in the playroom were not being cleaned according to the weekly protocol and may have served as fomites in the transmission of rotavirus.

FROM: Rogers M, Weinstock DM, Eagan J, Kiehn T, Armstrong D, Sepkowitz KA Rotavirus outbreak on a pediatric oncology floor: possible association with toys. Am J Infect Control 2000;28:378-380.

\section{Silver Alloy Urinary Catheters in Preventing UTIs}

Saint and coinvestigators from the University of Michigan conducted a study to assess the clinical and economic impact of using silver alloy urinary catheters in hospitalized patients. The decision model, performed from the healthcare payer's perspective, evaluated a simulated cohort of 1,000 hospitalized patients on general medical, surgical, urologic, and intensive care services requiring short-term urethral catheterization (2-10 days). They compared two catheterization strategies: silver alloy catheters and standard (noncoated) urinary catheters. Outcomes measured included the incidence of symptomatic UTI and bacteremia and direct medical costs.

In the base-case analysis, use of silver-coated catheters led to a $47 \%$ relative decrease in the incidence of symptomatic UTI from 30 to 16 cases per 1,000 patients (number needed to treat=74) and a $44 \%$ relative decrease in the incidence of bacteremia from 4.5 to 2.5 cases per 1,000 patients (number needed to treat $=500$ ) compared with standard catheters. Use of silver alloy catheters resulted in estimated cost savings of $\$ 4.09$ per patient compared with standard catheter use ( $\$ 20.87$ vs $\$ 16.78$ ). In a multivariate sensitivity analysis using Monte Carlo simulation, silver-coated catheters provided clinical benefits over standard catheters in all cases and cost savings in $84 \%$ of cases. The authors concluded that using silver alloy catheters in hospitalized patients requiring short-term urinary catheterization reduces the incidence of symptomatic UTI and bacteremia, and is likely to produce cost savings compared with standard catheters.

FROM: Saint S, Veenstra DL, Sullivan SD, Chenoweth $\mathrm{C}$, Fendrick AM. The potential clinical and economic benefits of silver alloy urinary catheters in preventing urinary tract infection. Arch Intern Med 2000;160:2670-2675.

\section{Ostomy as a Risk Factor for Post-traumatic Infection}

Primary repair for penetrating colonic injury is an acceptable practice in uncomplicated injuries, but it still is viewed with trepidation in high-risk patients. Dente and coinvestigators from Detroit Receiving Hospital, Wayne State University, Michigan, reviewed the records of 350 patients evaluated at an urban Level I trauma center for penetrating colonic injuries over an 8-year period (1989-1997). These included 33 stab and 317 gunshot wounds. Thirty-nine patients died within 48 hours. Of the remaining 311 patients, $78(25 \%)$ developed 152 infections. These infections were classified as traumatic or nosocomial in nature. Traumatic infections (46\%) included abdominal abscesses or peritonitis (28), wound infections (30), missile tract infections (8), and fistulas (4), whereas nosocomial infections (54\%) included pneumonia (25), bacteremia (25), urinary tract infections (17), miscellaneous (8), empyema (4), and sinusitis (3). Significance for analyses was set at $P<.05$.

Univariate analysis was performed to identify risk factors for the development of infections. The five most significant risk factors, using all infections as an outcome, were penetrating abdominal trauma index (PATI) greater than 30 , presence of an ostomy, multiple transfusions, Injury Severity Score (ISS) of 16 or greater, and Revised Trauma Score less than 7.8. All were highly significant $(P<.0001)$. Multivariate analysis with all infections as an outcome revealed that four of the five risk factors had independent effects, with the following significance: PATI greater than 30 , ISS of 16 or greater, ostomy, and multiple transfusions. Multivariate analysis for traumatic infections revealed only two of the above to be independent risk factors: presence of an ostomy $(P=.004)$ and a PATI greater than $30(P=.039)$, both of which can be considered local factors. Conversely, multivariate analysis of nosocomial infections revealed independent risk for the two other factors, both of which can be considered systemic factors: multiple transfusions $(P=.011)$ and ISS of 16 or greater $(P=.026)$.

The authors concluded that, although most of the above factors are beyond the control of the trauma surgeon, the creation of an ostomy is a clinical decision. The creation of an ostomy in high-risk patients does not protect them from septic complications and indeed may independently contribute to local abdominal infections.

FROM: Dente CJ, Tyburski J, Wilson RF, Collinge J, Steffes C, Carlin A. Ostomy as a risk factor for posttraumatic infection in penetrating colonic injuries: univariate and multivariate analyses. J Trauma 2000;49:628637. 